\title{
Novel thioredoxin targets in Dictyostelium discoideum identified by two-hybrid analysis: interactions of thioredoxin with elongation factor $1 \alpha$ and yeast alcohol dehydrogenase
}

\author{
Thomas Brodegger, Anja Stockmann, Jürgen \\ Oberstraß, Wolfgang Nellen and Hartmut \\ Follmann*
}

Departments of Biochemistry and Genetics, Natural Sciences Faculty, University of Kassel, D-34109 Kassel, Germany

\author{
${ }^{*}$ Corresponding author \\ e-mail: hfollman@uni-kassel.de
}

\begin{abstract}
Thioredoxins (Trx) are ubiquitous dicysteine proteins capable of modulating enzymes and other cellular targets through specific disulfide-dithiol redox changes. They are unique in that a large number of very diverse metabolic systems are addressed and redox-regulated in bacteria, animal, and plant cells, but the finite number of thioredoxin interaction partners is still unknown. Two-hybrid methodology should provide a rational way to establish thioredoxin functions in a given organism. We report a search for physiological target proteins of thioredoxin1 in the social amoeba Dictyostelium discoideum, which possesses three developmentally regulated thioredoxin genes, all of which lack functional characterisation. A two-hybrid approach identified at least seven bona fide thioredoxin partners, including oxidoreductases, proteins of the ribosomal translation apparatus, and the cytoskeletal protein filopodin. With the exception of ribonucleotide reductase, none of these systems had previously been linked to specific redox modulation. Molecular interactions in two of the new thioredoxin/target protein couples were verified by biochemical studies: (1) thioredoxin 1 and the abundant elongation factor $1 \alpha$ from $D$. discoideum form the mixed heterodisulfide characteristic of the thioredoxin mechanism of action; and (2) reduced thioredoxin, but not glutathione, strongly inhibits yeast alcohol dehydrogenase catalysis of ethanol oxidation.
\end{abstract}

Keywords: alcohol dehydrogenase; disulfide formation; elongation factors; enzyme regulation; thioredoxins; thioredoxin oligomers.

\section{Introduction}

The thioredoxin (Trx) family comprises a multitude of small proteins that contain a -W-C-G-P-C-K/R- hexapeptide sequence as the active site. In the reduced dithiol form (generated by NADPH: or ferredoxin:thioredoxin reductases) they are able to interact with enzymes, hormones, receptor proteins, etc., and to modulate their activity by reversible dithiol-disulfide exchange reactions with specific disulfide (cystine) sites in the target molecules (Follmann and Häberlein, 1995; Arnér and Holmgren, 2000; Follmann, 2000). The redox reaction passes through a mixed disulfide intermediate in which both partners are covalently linked through a disulfide bridge flanked by an $\mathrm{SH}$ group on either side. This dithiol-disulfide mechanism of metabolic regulation may equal the phosphorylation-dephosphorylation pathway in importance and universal distribution. Despite their small conserved structures, thioredoxins exhibit an extraordinary diversity of biochemical functions. More than 40 authentic thioredoxin-linked intra- and extracellular processes have been described in microbial, mammalian and plant cells and in cell organelles by biochemical studies (Follmann and Häberlein, 1995; Follmann, 2000). Most of these targets were originally identified by incidental observations, often guided by biochemical intuition. Many more interactions have been compiled by affinity chromatography and proteomics techniques (Balmer et al., 2003, 2004) but most of these potential new thioredoxin targets lack functional confirmation. The presence of multiple, differentially expressed thioredoxins in prokaryotic and eukaryotic cells further complicates the situation. Therefore, a reliable estimate of the finite number of biochemical systems specifically modulated by thioredoxins is not in sight.

We have reasoned (Brodegger et al., 2001) that the two-hybrid methodology for studying protein-protein interactions (Chien et al., 1991), now well established and productive, should be employed more comprehensively for specific detection of thioredoxin effects in both novel and known protein systems that have not yet been analysed for redox regulation. Only in a few studies, e.g., addressing mammalian cell proteins and a plant receptor kinase interaction, has the two-hybrid system been exploited for thioredoxin research (Makino et al., 1999; Yamanaka et al., 2000; Mazzurco et al., 2001).

Dictyostelium discoideum is an attractive, previously neglected object for such studies. Because of its highly unusual and complex life cycle, this lower eukaryote is a model organism for studying fundamental cellular and developmental processes (Kuspa et al., 1995; Maeda et al., 1997; Devreotes and Janetopoulos, 2003). Expression of a multigene family of thioredoxins has indeed been shown to be developmentally regulated (Wetterauer et al., 1992a,b). The amoebae contain at least three different proteins that exhibit only limited sequence homology $(30-40 \%)$ with other eukaryotic and bacterial thioredoxins. No physiologically relevant thioredoxin activities have yet been identified, and attempts to alter thioredoxin expression by antisense RNA constructs failed to show phenotypic changes (Brodegger, 2002). It is obvious that an alternative approach is needed to 
Table 1 Gene products identified by sequence analysis of positive clones obtained with $D$. discoideum thioredoxin1 in the bait vector of yeast two-hybrid assays.

\begin{tabular}{|c|c|c|c|}
\hline $\begin{array}{l}\text { Clone } \\
\text { Number }\end{array}$ & $\begin{array}{l}\text { Protein } \\
\text { number }\end{array}$ & Protein encoded (source) & Remarks \\
\hline 19 & O21042 & Cytochrome oxidase subunits $1 / 2(\mathrm{Dd})$ & \\
\hline 22 & & 40S ribosomal protein $\mathrm{S} 15$ (Dd) & 1 Cys \\
\hline 23 & gi7336 & Ribosomal acidic phosphoprotein PO (Dd) & \\
\hline 24 & & SSK571 regulatory protein (Dd) & \\
\hline 26 & Q03251 & SSI613 RNA binding protein (Dd) & 1 Cys \\
\hline 40 & P79732 & Ribonucleotide reductase R1 subunit (zebra fish) & a \\
\hline 42 & P18624 & Elongation factor $1 \alpha(\mathrm{Dd})$ & \\
\hline 45 & P90625 & RpgG homologue of ribosomal protein S3 (Dd) & \\
\hline 66 & $\mathrm{P} 00330 / 31$ & Alcohol dehydrogenase 1 and 2 (yeast) & b \\
\hline 72 & P54633 & Filopodin (homologue of talin) (Dd) & \\
\hline
\end{tabular}

The interactions with the proteins listed were missing in parallel experiments with a thioredoxin cysteine $\rightarrow$ serine mutant gene. Protein numbers refer to the SwissProt database. Dd, D. discoideum.

${ }^{a}$ Gene not yet documented in $D$. discoideum. Ribonucleotide reductases (class I) constitute a conserved enzyme family in $E$. coli and all eukaryotic cells.

${ }^{\mathrm{b}} \mathrm{ADH}$ genes and enzymes not yet characterised in $D$. discoideum. Yeast $A D H 1$ and $A D H 2$ genes are highly homologous to Dd sequence IIBPP1D02818 contained in the NCBI data bank.

understand better the significance and components of thioredoxin-directed redox regulation in this organism. We describe here an initiative to probe the interactions of $D$. discoideum thioredoxin1 with putative thioredoxin target proteins in a yeast two-hybrid system. Besides a known thioredoxin substrate, ribonucleotide reductase, at least six novel targets were found in this genetic approach. Two of them were subjected to biochemical analysis, confirming molecular interactions with thioredoxin in vitro by cross-linking and kinetic experiments.

\section{Results}

\section{Screening for thioredoxin interaction partners in a D. discoideum cDNA library}

An initial screen of a $D$. discoideum cDNA library with the yeast two-hybrid system containing the DdTrx1 gene in the bait vector, described in the Materials and methods section, produced 72 positive clones from a total of 272 primary transformants. Sequencing of the plasmids from positive clones transformed into $E$. coli and databank searches of the nucleotide and amino acid sequence levels identified 31 cysteine-containing proteins; cysteinefree sequences were discarded. These candidates exhibited e-values of $<0.1$ for sequence alignments, indicating biological relevance (Karlin and Altschul, 1990). The data included 12 genes coding for known $D$. discoideum proteins, five sequences homologous to yeast, and others homologous to plant and animal proteins. The identification of a eukaryotic ribonucleotide reductase large subunit, the earliest known thioredoxin redox partner in $E$. coli (Laurent et al., 1964), confirmed that the approach was functional.

The interaction of thioredoxins with specific target proteins requires a dicysteine site, essential for heterodisulfide formation in the majority of cases. To further validate the nature of target candidates we repeated the twohybrid screen using a double cysteine $\rightarrow$ serine mutant (C32S,C35S) of the DdTrx1 gene, which was obtained by site-directed mutagenesis. Barring the possibility of chaperone-like thiol-independent thioredoxin functions (Kern et al., 2003) such thioredoxin mutants have all other molecular domains unchanged, but in most cases they are poor functional interaction partners for thioredoxinresponsive target proteins, if at all. In fact, several transformants containing the double-mutant gene no longer grew on synthetic dropout (SD) medium, indicating that the positive interactions shown with the wild-type gene indeed depended on an intact thioredoxin dicysteine site.

This stringent selection left the 10 proteins (genes) listed in Table 1. Except for the known enzyme ribonucleotide reductase (clone 40), two monocysteine proteins (clones 22 and 26) and a candidate of uncertain nature (clone 24, probably related to clone 66), the remaining six positives are considered novel bona fide targets of thioredoxin1 in $D$. discoideum, and probably also in other organisms. The proteins are of cytoplasmic, ribosomal and mitochondrial origin, and might all be critical for the amoeba's developmental cycle. A cytoskeletal protein such as filopodin has not previously been linked to thioredoxin control, whereas oxidoreductases and proteins of the translation apparatus are per se not uncommon among thioredoxin targets. For biochemical confirmation of the presumed thioredoxin response, we chose two prominent proteins from Table 1, viz. alcohol dehydrogenase and the eukaryotic elongation factor $1 \alpha$.

\section{Inhibition of yeast alcohol dehydrogenase by thioredoxins in vitro}

The identification of clone 66 in the two-hybrid assay as an alcohol dehydrogenase (ADH) gene came as a surprise, because the thiol and disulfide status of the eight cysteine residues present in ADH under the influence of thiol reagents and reductants has been studied for decades (Wallenfels and Sund, 1957; Bühner and Sund, 1969; Belke et al., 1974). The requirement for a low amount $(\leq 2 \mathrm{~mm})$ of reduced glutathione (GSH) in standard ADH activity assays is well known (Bergmeyer, 1974), but to the best of our knowledge thioredoxin was 

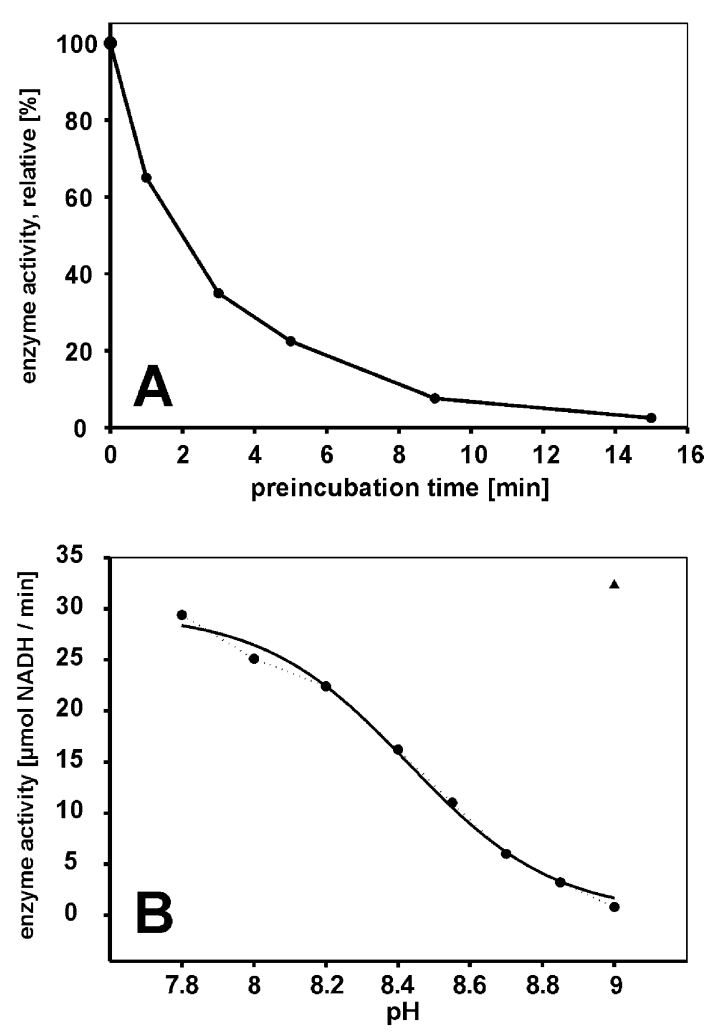

Figure 1 Inhibition of yeast alcohol dehydrogenase incubated with $0.8 \mu \mathrm{M}$ thioredoxin (10 $\mu \mathrm{g}$ per assay).

(A) Time course (at $\mathrm{pH}$ 9). (B) $\mathrm{pH}$ dependence (incubation for $15 \mathrm{~min})$. The symbol in the upper right corner represents the enzyme activity in the absence of thioredoxin. Enzyme activity was determined at $\mathrm{pH} 9$ and $25^{\circ} \mathrm{C}$, measuring $\mathrm{NADH}$ formation at $340 \mathrm{~nm}$.

never included. Unfortunately, the amoebic enzyme has not been described to date. Alignment of available DNA sequences showed $98 \%$ homology with yeast $A D H 1$ and $A D H 2$ genes, suggesting that the enzymes of $D$. discoideum and Saccharomyces cerevisiae are highly conserved. (The possibility cannot be ruled out, however, that this cDNA was actually a yeast contaminant in the library.) We could readily detect ADH activity in $D$. discoideum cell extracts, but were unsuccessful in obtaining an enzyme fraction suitable for kinetic experiments by standard purification steps. Therefore, yeast ADH was used for in vitro activity measurements in the presence of various thioredoxins.

The addition of thioredoxins in the disulfide form (i.e., as isolated) to standard ADH assays containing reduced glutathione did not affect enzyme activity. However, reduced thioredoxin $(0.8 \mu \mathrm{M})$ strongly inhibited $\mathrm{ADH}$ catalysed ethanol oxidation by $\mathrm{NAD}^{+}$at alkaline $\mathrm{pH}$ (Figure 1). Essentially identical effects were produced by the thioredoxins obtained from $E$. coli, yeast and $D$. discoideum. Under defined conditions, the reduction of thioredoxins was achieved throughout these experiments by pretreatment with dithiothreitol followed by gel filtration for removal of the low-molecular-weight reductant, or in situ in the presence of a dithiothreitol concentration not affecting the enzyme by itself ( $\leq 3 \mathrm{mM})$. Such in vitro conditions reliably mimic the action of NADPH:thioredoxin reductases, which in heterologous protein systems show variable specificity towards thioredoxin substrates.
Almost complete inhibition was reached after 10-15 min of incubation of $\mathrm{ADH}$ and reduced $\mathrm{Trx}$ at $\mathrm{pH} 9$ (Figure 1A). The $\mathrm{pH}$ dependence curve (Figure 1B) suggests that an ionisable species with a $\mathrm{pK}_{\mathrm{a}}$ value of approximately 8.4 is involved, most likely a cysteine residue. Inhibition was also observed in the presence of a large excess (>5 mM) of the strong reductant dithiothreitol without thioredoxin, but not with up to $20 \mathrm{~mm}$ GSH. ADH catalysis of the reverse reaction, i.e., ethanol formation from acetaldehyde and $\mathrm{NADH}$ at $\mathrm{pH} 7$, is also inhibited by thiols. However, in contrast to the thioredoxin-specific behaviour described above, $2.5 \mathrm{~mm}$ dithiothreitol or $5 \mathrm{~mm}$ GSH alone produced strong inhibition, while reduced thioredoxin alone or in the presence of the other reductants had no effect. When dithiothreitol (5 mM) and samples of reduced thioredoxin were combined with $\mathrm{ADH}$ from horse liver under the above ethanol oxidation conditions, the mammalian enzyme did not respond with any change in activity. Further mechanistic studies were not within the scope of our present work, but a molecular model and the physiological significance of thioredoxin action upon yeast $A D H$ are addressed in the discussion section.

\section{Mixed disulfide formation between elongation factor $1 \alpha$ and thioredoxin1}

Eukaryotic elongation factor $1 \alpha(E F 1 \alpha)$ is an abundant cellular protein that, besides its function in ribosomal protein synthesis, assumes multiple physiological activities (Condeelis, 1995). It does not lend itself to a simple activity assay of thioredoxin interactions in vitro. However, the monomeric structure of the $D$. discoideum protein which contains only five cysteine residues (Yang et al., 1990), two of which are likely arranged in an accessible dicysteine loop, should permit the observation of specific binding to a thioredoxin through a covalent heterodisulfide, which is the common intermediate in thioredoxindependent processes. As native heterodisulfide intermediates are kinetically labile and difficult to observe, if at all, this approach requires that one of the interacting cysteines is replaced by a redox-inactive serine residue to produce a more stable heterodimer.

We therefore constructed the cysteine $\rightarrow$ serine C35S mutant of $D$. discoideum thioredoxin1 and combined it with recombinant $D$. discoideum $\mathrm{EF} 1 \alpha$. Both proteins were overexpressed in $E$. coli and were tagged with a Strep-tag and a C-terminal His-tag, respectively, to allow immunological detection. Formation of covalently linked heterodimers should reveal a new protein band at a molecular mass of $14+55=69 \mathrm{kDa}$ on non-reducing polyacrylamide gels.

Direct incubation of the purified EF $1 \alpha$ and Trx 1 species under various conditions failed to generate the expected $69-\mathrm{kDa}$ species. The failure may have been due to limiting protein concentrations $(10 \mu \mathrm{M})$, and in particular to unexpected intermolecular oxidation of the thioredoxin mutant, producing a series of oligomeric homodisulfides (Figure 3 , see below), competing with and preventing heterodisulfide production.

As an alternative, formation of $\mathrm{EF} 1 \alpha-\operatorname{Tr} x 1$ covalent complexes was probed by affinity chromatography of the elongation factor on columns containing Strep-tagged 

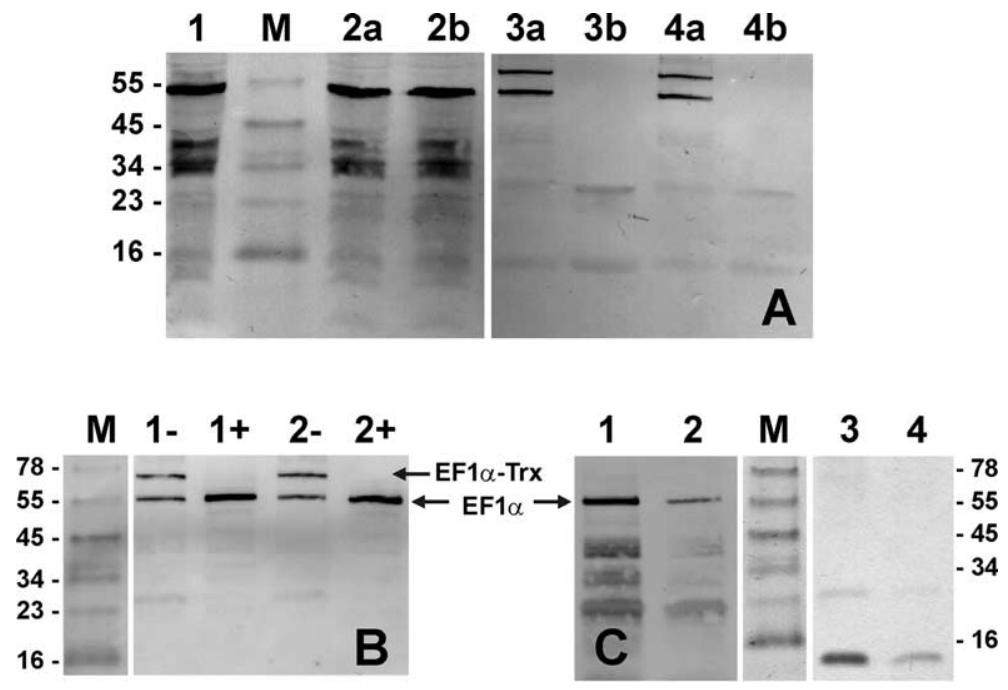

Figure 2 Formation of EF1 $\alpha$-Trx 1 heterodimers by affinity chromatography of His-tagged elongation factor on Strep-tagged Trx 1 species bound to Strep-Tactin-Sepharose.

Fractions were analysed by SDS polyacrylamide electrophoresis and proteins detected on Western blots. Marker proteins (M): bovine serum albumin (BSA; $78 \mathrm{kDa})$, glutamic dehydrogenase $(55 \mathrm{kDa})$, alcohol dehydrogenase (45 kDa), carbonic anhydrase (34 kDa), myoglobin $(23 \mathrm{kDa})$, lysozyme $(16 \mathrm{kDa})$, and aprotinin $(6 \mathrm{kDa})$.

(A) Interaction of EF1 $\alpha$ with immobilised Trx1-C35S (a) or Trx1-C32S,C35S (b). Lane 1, cell-free extract containing overexpressed $\mathrm{EF} 1 \alpha$ before chromatography. Lanes 2a,b, flow-through fractions. Lanes $3 a, b, 4 a, b$, eluate fractions 3 and 4 obtained by elution with dethiobiotin. (B) Reversibility of heterodimer formation. Lanes 1- and 2-, eluate fractions 3 and 4 (as in lanes 3a and 4a, above) kept in non-reducing conditions. Lanes $1+$ and $2+$, the same fractions reduced in the presence of dithiothreitol. (C) Interaction between EF1 $\alpha$ and immobilised wild-type Trx1, analysed as in panel (A). Lanes 1 and 2 (left panel), eluate fractions 3 and 4 as above, detection on Western blots. Lanes 3 and 4 (right panel), the same fractions, detection by protein staining.

Trx1-C35S bound to Strep-Tactin-Sepharose. The system was adjusted with diamide-containing buffer to maintain conditions suitable for disulfide formation, and a cell-free bacterial extract containing overexpressed His-tagged EF1 $\alpha$ was applied. After washing the column with redox-neutral buffer, Strep-tagged proteins were dissociated by elution with $2.5 \mathrm{~mm}$ dethiobiotin. The fractions were subjected to SDS gel electrophoresis and analysed with anti-His antibodies on Western blots. As shown in Figure 2A, unbound elongation factor passed through the column in the wash buffer, but elution released a protein with a molecular mass of $69 \mathrm{kDa}$, in addition to free $\mathrm{EF} 1 \alpha$, in eluate fractions 3 and 4 . Parallel runs on columns containing the redox-inactive double-



Figure 3 Reversible oligomerisation of Strep-tagged $D$. discoideum thioredoxins (10- $\mu \mathrm{g}$ samples).

Lanes 1-3: in oxidising buffer containing $5 \mathrm{mM}$ diamide; lanes 4-6: after reduction of the same samples with $10 \mathrm{~mm}$ dithiothreitol for $30 \mathrm{~min}$. Lanes 1 and 4: wild-type Trx1; lanes 2 and 5: Trx1-C35S; lanes 3 and 6: Trx1-C32S,C35S double mutant. Electrophoresis was carried out on SDS polyacrylamide gels in redox-neutral Tris-Tricine buffer and the proteins were detected on Western blots. Marker proteins (M) as in Figure 2. The band at $22 \mathrm{kDa}$ represents residual $E$. coli carboxybiotin carrier protein (BCCP) co-eluted during Strep-Tactin affinity chromatography. serine mutant of Trx1 (C32S,C35S) immobilised on StrepTactin-Sepharose did not reveal any EF1 $\alpha$ species of $>55 \mathrm{kDa}$. Proof for the presence of an intermolecular disulfide bond in the presumed $69-\mathrm{kDa}$ heterodisulfide was provided by reduction of the eluate fractions with $10 \mathrm{~mm}$ dithiothreitol, which regenerated the free elongation factor of $55 \mathrm{kDa}$ (Figure 2B).

An attempt was made to demonstrate disulfide formation with wild-type thioredoxin1. As expected, a TrxEF1 $\alpha$ heterodimer could not be observed in chromatography runs on a Strep-Tactin-Sepharose column loaded with the Strep-tagged wild-type protein (Figure 2C). However, in this case column fractions 3 and 4 (see above) contained both $\mathrm{EF} 1 \alpha(55 \mathrm{kDa})$ and thioredoxin $(14 \mathrm{kDa})$, i.e., the breakdown products of a native heterodimer intermediate. Control runs showed that EF1a alone was not retarded unspecifically on Strep-TactinSepharose lacking bound Trx1, and thus could not have been eluted in these particular fractions (Figure 2C, lanes 1 and 2), nor was free thioredoxin (lanes 3 and 4) found in any other eluate fraction. Taken together, these observations confirm that the two proteins interact by way of the mixed disulfide mechanism characteristic of thioredoxin-target protein couples.

\section{Oligomers of $\boldsymbol{D}$. discoideum thioredoxin1}

Thioredoxin1 of $D$. discoideum contains a third cysteine at position 10, apparently not engaged in physiological redox functions, in addition to the active-site cysteines 32 and 35 . Mutant protein C35S thus has two single cysteines 20 amino acids apart. Under oxidising conditions on SDS polyacrylamide electrophoresis gels we observed a series of disulfide-linked oligomers in the 
range from 14 to $>120 \mathrm{kDa}$ (Figure 3 ), dominated by the 28-kDa dimer. Higher oligomers could be distinguished up to the decamer. Wild-type Trx1 showed the same, somewhat less pronounced behaviour, whereas a dimer (i.e., the only possible product) was formed from the cysteine $\rightarrow$ serine double mutant in oxidising media. Reduction by dithiothreitol produced the monomeric thioredoxin species.

Such facile oligomerisation has not been described for any other thioredoxin, including chloroplast thioredoxin $f$, which also bears a third cysteine (albeit near the C-terminus) and has been studied in heterodimer formation with fructose-bis-phosphatase (Balmer and Schürmann, 2001). The protein ladder of $D$. discoideum Trx1-C35S oligomers described here is of practical use as a molecular-mass marker kit for SDS gel electrophoresis (Stockmann, 2002).

\section{Discussion}

Despite impressive advances in the identification of potential thioredoxin functions, which exceed 100 (Balmer et al., 2003, 2004), it remains very difficult to establish a picture of the physiological relevance of thioredoxin action in any given organism for the following reasons. (1) Eukaryotic cells contain multiple thioredoxin genes, which may be differentially expressed. (2) The vast number of cellular processes that have been linked to thioredoxin/thioredoxin reductase redox control by various in vitro strategies makes it highly unlikely, if not impossible, that all these interactions operate simultaneously and ubiquitously. (3) The conserved molecular structure of thioredoxins frequently allows protein interactions among heterologous partners in vitro that are not identical to those observed in homologous combinations of thioredoxins and target proteins derived from the same organism. (4) Except in the case of enzymes, peptide hormones, etc., activity assays for direct functional proof of thioredoxin-target protein interaction may be difficult or lacking.

Despite such obstacles, it is still imperative to substantiate genetic and proteomics screening results by robust biochemical information wherever possible. Of the candidate thioredoxin targets in Table 1, we selected an enzyme, $\mathrm{ADH}$, and a ribosomal factor, $\mathrm{EF} 1 \alpha$, for confirmation of thioredoxin effects, because they are involved in well-defined metabolic functions, are of monomeric protein structure and are amenable to activity determination.

\section{Thioredoxin interaction with alcohol dehydrogenase}

An interaction between thioredoxin and alcohol dehydrogenases has not been considered previously but is per se easily reconcilable with $\mathrm{ADH}$ structures. Both yeast and mammalian enzymes possess extra cysteine residues in addition to the six cysteines required to chelate the catalytic and structural zinc ions (Bühner and Sund, 1969) but their individual roles have not been established. $A D H 1$ and $A D H 2$ are the two main isozymes expressed in yeast. $A D H 2$, which preferentially catalyses the conversion of ethanol to acetaldehyde (i.e., the thioredoxin- inhibited reaction), contains two cysteines in the C-terminal half (Cys259, Cys276) separated by 16 amino acids (Russell et al., 1983), resembling thioredoxin target sequences found in other redox-regulated enzymes (e.g., Marcus and Harrsch, 1990). Although their redox state in the active enzyme and the three-dimensional structure of yeast $A D H$, which resists crystallisation, are unknown, we expected an involvement of specific cysteines in the thioredoxin-induced activity change reported here (cf. Figure 1B). A chaperone-like function of thioredoxin independent of cysteine residues (Kern et al., 2003) appears unlikely in this case. In contrast, in horse liver $A D H$, which was not inhibited by thioredoxin, cysteine residues not engaged in zinc binding assume completely different positions in the polypeptide sequence (Jörnvall, 1977). This is in accord with the different structures and physiological functions of ADH in yeast and mammals (Jörnvall, 1977; Deltour et al., 1999). More detailed functional analysis of the various cysteines is desirable.

A rationale for the inhibition of ethanol oxidation in yeast by reduced thioredoxins (including the homologous yeast protein) may lie in yeast metabolism. Under anaerobic conditions, with an excess of reducing equivalents and thioredoxin in the reduced state, the reduction of acetaldehyde by $\mathrm{NADH}$, required for the regeneration of $\mathrm{NAD}^{+}$, is the predominant direction and would clearly be favoured by specific suppression of the reverse reaction. In contrast, as $\mathrm{pH}$ decreases and ethanol concentration rises, it might be of advantage to cells if the inhibition of ethanol oxidation was relieved and instead acetaldehyde reduction suppressed, albeit in an unspecific fashion and at much higher (probably non-physiological) thiol concentrations. Yeast cultures carrying mutant $\mathrm{ADH}$ and thioredoxin genes should be an obvious system to verify these correlations. Alcohol dehydrogenase and its metabolic role in the amoebae also remain to be characterised.

Very recently, $\mathrm{ADH}$ has been listed among numerous potential thioredoxin targets identified by affinity chromatography of pea leaf mitochondrial extracts (Balmer et al., 2004). No activity data or other details were communicated. The sequence of yeast mitochondrial ADH differs strongly from yeast $\mathrm{ADH} 1$ and $\mathrm{ADH} 2$ discussed above.

\section{Elongation factor $1 \alpha$ as thioredoxin target}

Disulfide-dithiol redox reactions between thioredoxins and target proteins include short-lived mixed disulfide intermediates in which the two partners are covalently linked through a disulfide bridge (Follmann and Häberlein, 1995; Arnér and Holmgren, 2000). Using cysteine $\rightarrow$ serine mutant proteins to prevent rapid breakdown of the mixed disulfide heterodimer, stable cross-links have been generated in a number of thioredoxin-regulated protein systems, in particular for analysis of chloroplast enzymethioredoxin interactions (Balmer and Schürmann, 2001; Goyer et al., 2001). The experiments combining EF1 $\alpha$ and $\operatorname{Tr} x 1$ of $D$. discoideum demonstrate that they, too, interact in a thioredoxin-specific, physiologically relevant fashion.

A structural model of a putative thioredoxin site in elongation factor EF1 $\alpha$ has been put forward (Brodegger, 
Table 2 PCR primers used for amplification and site-directed construction of thioredoxin1 and cysteine $\rightarrow$ serine mutants, respectively.

\begin{tabular}{ll}
\hline Primer number and sequence & Required for \\
\hline 1: 5'-GAACGAGCTCCATGGCCAATAGAGTAATTCATG-3' & Trx1 amplification \\
2: 5'-CGCGGATCCTTATTTGTTGCTTCTAGAGTACTTC- 3' & Trx1 amplification \\
3: 5'-CTTTAGTGCTGTATGGTCTGGCCCATCTAGAGCAATATCTCC-3' & Ser,Ser double mutant \\
4: 5'-GGAGATATTGCTCTA-GATGGGCCAGACCATACAGCACTAAAG-3' & Ser,Ser double mutant \\
5: 5'-CTTAGTGCTGTATGGTGT-GGCCCATCTAGAGCAATATCTCC-3' & C35S mutant \\
6: 5'-GGAGATATTGCTCTA-GATGGGCCACACCATACAGCACTAAAG-3' & C35S mutant \\
\hline
\end{tabular}

Nucleotides in bold face produce Cys $\rightarrow$ Ser codon changes. Underlined nucleotides indicate a silent mutation in the active-site glycine codon for deletion of an Avall restriction site.

2002). Compared with prokaryotic EF-Tu, the highly conserved eukaryotic proteins of fungi and animal cells possess an insertion of 13 amino acids, including two cysteines. Molecular modelling based on the structure of EF-Tu from Thermus aquaticus (Nissen et al., 1999) suggests that the eukaryotic sequence containing Cys354 and Cys361 forms a surface loop accessible for thioredoxin interaction. The situation closely resembles the structure difference between thioredoxin-independent cytosolic fructose-bis-phosphatases and the thioredoxin-regulated chloroplast enzyme, which displays a 17-amino-acid insertion sequence containing extra cysteines (Marcus and Harrsch, 1990; Villeret et al., 1995). Site-directed mutagenesis of the cysteines in EF1 $\alpha$ is a means to test that model. If true, bacterial EF-Tu should not respond to thioredoxins. Its entry in a list of potential Trx targets (Balmer et al., 2004) remains unsubstantiated at present.

The physiological significance of thioredoxin-mediated redox regulation of EF1 $\alpha$ is not easy to assess in view of its multiple cellular functions (Condeelis, 1995). It is well established that the ribosomal translation rate is stimulated by reducing conditions, including the thioredoxin system (Hunt et al., 1983), but not much is known about which of the many participating proteins are affected. Note that other ribosomal proteins are listed in Table 1 besides EF1 $\alpha$. In Dictyostelium, in which EF1 $\alpha$ also serves an actin-binding function (Yang et al., 1990), an involvement in the redox regulation of cytoskeleton and differentiation processes is another attractive hypothesis.

In conclusion, our two-hybrid assays combined with biochemical experiments have revealed new details of thioredoxin interactions in the amoeba Dictyostelium, and in two well-known proteins not previously recognised as thioredoxin-linked. The genetic approach appears to produce more meaningful information about the general dimensions and specificity of thioredoxin regulation in biology than affinity screening protocols not accompanied by functional studies. This analysis needs to be extended to the other thioredoxins expressed in $D$. discoideum. Furthermore, it is evident that thioredoxin control of ribosomal and cytoskeletal proteins in this and other organisms deserve systematic study.

\section{Materials and methods}

\section{Chemicals and proteins}

All chemicals and reagents were of the highest quality available. Alcohol dehydrogenase from yeast and horse liver were prod- ucts of Merck (Darmstadt, Germany) and Sigma (Deisenhofen, Germany), respectively. Thioredoxin from E. coli, and sheep antibodies directed against it were obtained from IMCO (Stockholm, Sweden). Yeast thioredoxin was prepared according to the published procedure (Porqué et al., 1970). If required, samples of reduced thioredoxin were generated in deaerated solutions by incubation of the oxidised protein with $10 \mathrm{~mm}$ dithiothreitol and subsequent removal of excess reductant by passage over a HiTrap desalting column (Pharmacia, Freiburg, Germany). Thioredoxin1 from $D$. discoideum was overexpressed in $E$. coli cells. For this purpose the cDNA sequence of $\operatorname{Tr} x 1$ was amplified by polymerase chain reaction (PCR) using primers 1 and 2 (Table 2) and cloned into the $\mathrm{NcOl}$ and Bamll restriction sites of the expression vector pET15b (Novagen, Madison, USA). The protein was extracted and purified to apparent homogeneity following a standard thioredoxin protocol including heat $\left(70^{\circ} \mathrm{C}\right)$ treatment, gel filtration on Sephadex G75, and ion exchange chromatography on UnoQ2 and MonoS columns. The preparation of cysteine $\rightarrow$ serine mutant thioredoxins is described below.

\section{General methods}

Protein was determined using Bradford dye reagent (Bio-Rad, Munich, Germany). The activity of thioredoxins was assayed spectrophotometrically by following thioredoxin-stimulated NADPH oxidation catalysed by NADP malate dehydrogenase (NADP-MDH) from spinach chloroplasts (Jacquot et al., 1978). SDS gel electrophoreses were run on $10 \%$ polyacrylamide gels in a Tris-Tricine buffer system (Schägger and von Jagow, 1987), with or without the addition of $50 \mathrm{~mm}$ dithiothreitol. Proteins were stained with Coomassie brilliant blue G-250. Proteins containing Strep-tag or His-tag sequences were visualised on Western blots by standard procedures, using Strep-Tactin alkaline phosphatase (IBA, Göttingen, Germany) and anti-His mouse antibodies (Amersham-Pharmacia, Braunschweig, Germany), respectively, for detection. Prestained molecular weight marker proteins were from Novex (Frankfurt, Germany).

\section{Site-directed mutagenesis}

The wild-type Trx1 gene was cloned into the BamHI and Sacl restriction sites of plasmid pGem3Z (Promega, Mannheim, Germany) as template. Mutagenesis for the replacement of cysteine by serine residues, accompanied by deletion of an Avall restriction sequence in the active site, was carried out using a standard PCR protocol using the primers listed in Table 2. Reaction mixtures contained $5 \mu \mathrm{l}$ of buffer $(0.2 \mathrm{M}$ Tris- $\mathrm{HCl}, \mathrm{pH} 8.8$, containing $0.1 \mathrm{M}$ ammonium sulfate, $20 \mathrm{~mm}$ magnesium sulfate, $1 \%$ Triton $\mathrm{X}-100$ and $1 \mathrm{mg} / \mathrm{ml}$ BSA), 5-50 ng of template DNA, $125 \mathrm{ng}$ of primer (each), $1 \mu \mathrm{l}$ of dNTP mix (2 $\mu$ mol each) and $1 \mu \mathrm{l}$ of Taq DNA polymerase, and were adjusted to $50 \mu \mathrm{l}$ with water. PCR was run through 20 cycles $\left(30 \mathrm{~s}\right.$ at $95^{\circ} \mathrm{C}, 1 \mathrm{~min}$ at $55^{\circ} \mathrm{C}, 2 \mathrm{~min}$ per kilobase of the template at $72^{\circ} \mathrm{C}$ ). The mixture was then treated with restriction enzyme Dpnl $(10 \mathrm{U} / \mu \mathrm{l})$ to digest methylated template DNA $\left(1 \mathrm{~h}\right.$ at $\left.37^{\circ} \mathrm{C}\right)$ before it was heated to 
$65^{\circ} \mathrm{C}$ for $20 \mathrm{~min}$ to inactivate the enzyme. The mutagenised thioredoxin sequences were transformed into $E$. coli strain XL1-Blue (Stratagene, Amsterdam, The Netherlands) by the standard procedure (Dagert and Ehrlich, 1979). Successful mutagenesis was demonstrated by resistance of the plasmids towards Avall digestion on agarose electrophoresis gels.

\section{Two-hybrid assays}

The two-hybrid system was obtained from Clontech Laboratories (Palo Alto, USA). A D. discoideum cDNA library cloned into the PACT2 vector was kindly provided by Dr. A. Kuspa (Baylor College, Houston, Texas, USA). Assays were carried our in Saccharomyces cerevisiae strain Y190 with plasmids pAS2 as the bait and pACT2 as the prey vector. Thioredoxin1 cDNA was cloned into the BamH1 and Ncol sites of pAS2 by the above PCR procedure (primers 1 and 2). Transformation procedures were carried out as described by Gietz and Woods (1995). The selection for positive double-transformation occurred on synthetic dropout (SD) medium without leucine, histidine and tryptophan. $\beta$-Galactosidase colony-lift filter assays followed the protocol from Clontech.

Sequencing of plasmids from positive yeast clones, retransformed in E. coli DH5 $\alpha$ (Promega) and purified over Nucleobond AX20 columns (Macherey-Nagel, Düren, Germany) was carried out using an ABI Prism BigDye Terminator Sequencing Kit (Perkin Elmer-Applied Biosciences, Weiterstadt, Germany). The sequences were aligned versus the $D$. discoideum (http://dictybase.org) and NCBI databases (http://www.ncbi.nlm.nih.gov/ BLAST/).

\section{Preparation of elongation factor $1 \alpha$}

The EF1 $\alpha$ gene from $D$. discoideum was inserted into $\mathrm{pET} 15 \mathrm{~b}$ containing a His-tag sequence (from Novagen) and overexpressed in E. coli strain BL21. Cell-free extracts were passed over a His-Trap column equilibrated with nickel ions, and the protein was eluted with $50 \mathrm{mM}$ Tris buffer, $\mathrm{pH} 7.9$, containing $0.3 \mathrm{M}$ imidazole and $0.5 \mathrm{M} \mathrm{NaCl}$. Elution was followed on SDS polyacrylamide electrophoresis gels and detection with anti-His antibodies on Western blots. Fractions containing EF1 $\alpha(55 \mathrm{kDa})$ could best be concentrated using Aquacide II (from Calbiochem) and stored at $4{ }^{\circ} \mathrm{C}$ for short periods of time. A yield of $320 \mu \mathrm{g}$ of homogeneous protein was obtained from $500 \mathrm{ml}$ of bacterial culture.

\section{Preparation of mutant thioredoxins Trx1-C35S and Trx1-C32S,C35S}

Cysteine $\rightarrow$ serine mutant thioredoxins were obtained by sitedirected mutagenesis (primers 3-6, see Table 2). Mutant genes containing a C-terminal Strep-tag sequence (IBA, Göttingen, Germany) were cloned into the above $\mathrm{pET} 15 \mathrm{~b}$ vector lacking the His-tag sequence, and were overexpressed in E. coli BL21. Cells from a $100-\mathrm{ml}$ culture were suspended in $1 \mathrm{ml}$ of $100 \mathrm{mM}$ Tris buffer, $\mathrm{pH} 8.0$, containing $150 \mathrm{~mm} \mathrm{NaCl}, 1 \mathrm{~mm}$ EDTA and $5 \mathrm{~mm}$ dithiothreitol, and were lysed by sonication. Avidin $(20 \mu \mathrm{g})$ was added to block biotinylated proteins, and after $30 \mathrm{~min}$ in the cold the lysate was centrifuged at $14000 \mathrm{~g}$ for $30 \mathrm{~min}$. The clear supernatant was applied to a column of Strep-Tactin-Sepharose equilibrated in the above buffer, and unbound proteins were removed with five $1-\mathrm{ml}$ volumes of buffer. Bound proteins carrying the Strep-tag were then obtained by elution with six 0.5 $\mathrm{ml}$ volumes of $2.5 \mathrm{~mm}$ dethiobiotin in the same buffer. The fractions were analysed by SDS gel electrophoresis and Western blotting, and in enzyme activation assays. Trx1(C35S)-Strep-tag was obtained in $3.2 \mathrm{mg}$ yield, and the double-serine mutant in
$2.1 \mathrm{mg}$ yield. The proteins were $>90 \%$ pure, and in contrast to wild-type $\operatorname{Tr} x 1$ they were totally inactive towards NADP-MDH.

\section{Interaction of elongation factor EF1 $\alpha$ with thioredoxins}

A preparation of C35S mutant Trx1-Strep-tag was bound to a column of Strep-Tactin-Sepharose as described above, and unbound proteins were washed out. Dithiothreitol in the column buffer was then replaced by $5 \mathrm{~mm}$ diamide, and subsequent steps were carried out in a Tris/ $\mathrm{NaCl} / \mathrm{EDTA}$ buffer free of oxidising or reducing agents. A cell-free extract was obtained from a 250-ml bacterial culture overexpressing His-tagged EF1 $\alpha$ and was applied to the column. After removal of unspecific proteins, fractions were obtained by specific elution with $2.5 \mathrm{~mm}$ dethiobiotin in the redox-neutral buffer and analysed on SDS electrophoresis gels in dithiothreitol-free media (cf. Figure 2).

\section{Alcohol dehydrogenase assay}

Kinetic assays of alcohol dehydrogenases were carried out at $25^{\circ} \mathrm{C}$ in analogy to standard procedures (Bergmeyer, 1974). Reduced glutathione required in the assays to maintain full ADH activity ( $\leq 2 \mathrm{~mm}$ ) could be replaced by dithiothreitol. For ethanol oxidation, assays contained, in a total volume of $1 \mathrm{ml}, 300 \mathrm{~mm}$ ethanol, $100 \mathrm{~mm}$ semicarbazide hydrochloride, $1.5 \mathrm{mM} \mathrm{NAD}^{+}$ and 0.01-0.09 U ADH in $100 \mathrm{~mm}$ pyrophosphate-glycine buffer, $\mathrm{pH}$ 9.0. Acetaldehyde reduction assays contained $10 \mathrm{~mm}$ acetaldehyde (freshly distilled), $1 \mathrm{~mm} \mathrm{NADH}$ and $0.09 \mathrm{U}$ ADH in $200 \mathrm{~mm}$ potassium phosphate buffer, $\mathrm{pH}$ 7.0. Preincubation of enzyme samples with $10 \mu \mathrm{g}$ of reduced thioredoxin, generated by treatment with dithiothreitol, is described in the text (see above). The reactions were started by the addition of substrate and coenzyme, and product formation was monitored spectrophotometrically at $340 \mathrm{~nm}$.

\section{Acknowledgements}

We thank the Otto-Braun-Foundation, Melsungen and Kassel, for a stipend (to T.B.), and Drs. Ingo Häberlein (ESPE Dental, Seefeld, Germany) and Birgit Wetterauer (Zoologisches Institut, Universität München) for stimulating discussions and advice.

\section{References}

Arnér, E.S.J., and Holmgren, A. (2000). Physiological functions of thioredoxin and thioredoxin reductase. Eur. J. Biochem. 267, 6102-6109.

Balmer, Y., and Schürmann, P. (2001). Heterodimer formation between thioredoxin $\mathrm{f}$ and fructose-1,6-bisphosphatase from spinach chloroplasts. FEBS Lett. 492, 58-61.

Balmer, Y., Koller, A., del Val, G., Manieri, W., Schürmann, P., and Buchanan, B.B. (2003). Proteomics gives insight into the regulatory function of chloroplast thioredoxins. Proc. Natl. Acad. Sci. USA 100, 370-375.

Balmer, Y., Vensel, W.H., Tanaka, C.K., Hurkman, W.J., Gelhaye, E., Rouhier, N. Jacquot, J.P., Manier, W., Schürmann, P., Droux, M., and Buchanan, B.B. (2004). Thioredoxin links redox to the regulation of fundamental processes of plant mitochondria. Proc. Natl. Acad. Sci. USA 101, 2642-2647.

Belke, C.J., Chin, C.C.Q., and Wold, F. (1974). Effect of pH on the reactivity of the active-site sulfhydryl groups in yeast alcohol dehydrogenase. Biochemistry 13, 3418-3420.

Bergmeyer, H.U. (1974). Methoden der enzymatischen Analyse. 3rd Edition (Weinheim, Germany: Verlag Chemie), pp. 458-459, 1552-1554.

Brodegger, T. (2002). Molekularbiologische Funktionsanalysen 
von Thioredoxinen aus Dictyostelium discoideum. $\mathrm{PhD}$ thesis (Kassel, Germany: University of Kassel).

Brodegger, T., Stockmann, A., Nellen, W., Follmann, H., and Oberstraß, J. (2001). Identification of new thioredoxin interaction partners by the two-hybrid system. Biol. Chem. 382, S167.

Bühner, M., and Sund, H. (1969). Yeast alcohol dehydrogenase. $\mathrm{SH}$ groups, disulfide groups, quaternary structure, and reactivation by reductive cleavage of disulfide groups. Eur. J. Biochem. 11, 73-79.

Chien, C., Bartel, P.L., Sternglanz, R., and Fields, S. (1991). The two-hybrid system: a method to identify genes for proteins that interact with a protein of interest. Proc. Natl. Acad. Sci. USA 88, 9578-9582.

Condeelis, J. (1995). Elongation factor $1 \alpha$, translation and the cytoskeleton. Trends Biochem. Sci. 20, 169-170.

Dagert, M., and Ehrlich, S.D. (1979). Prolonged incubation in calcium chloride improves the competence of Escherichia coli cells. Gene 6, 23-28.

Deltour, L., Foglio, M.H., and Duester, G. (1999). Metabolic deficiencies in Adh null mutant mice. Overlapping roles of Adh1 and Adh4 in ethanol clearance and metabolism of retinol. J. Biol. Chem. 274, 16796-16801.

Devreotes, P., and Janetopolous, C. (2003). Eukaryotic chemotaxis: distinctions between directional sensing and polarization. J. Biol. Chem. 278, 20445-20448.

Follmann, H. (2000). Light-dark and thioredoxin-mediated metabolic redox control in plant cells. In: The Redox State and Circadian Rhythms, T. Vanden Driessche, ed. (Dordrecht, The Netherlands: Kluwer Academic Publishers), pp. 59-83.

Follmann, H., and Häberlein, I. (1995). Thioredoxins: universal, yet specific thiol-disulfide redox cofactors. BioFactors 5, 147-156.

Gietz, R.D., and Woods, R.A. (1995). Transforming yeast with DNA. Methods Mol. Cell. Biol. 5, 255-269.

Goyer, A., Decottignies, P., Issakidis-Bourguet, E., and MiginiacMaslow, M. (2001). Sites of interaction of thioredoxin with sorghum NADP malate dehydrogenase. FEBS Lett. 505, 405-408.

Hunt, T., Herbert, P., Campbell, E.A., Delidakis, C., and Jackson, R.J. (1983). The use of affinity chromatography reveals a requirement for $\mathrm{NADPH}$, thioredoxin, and thioredoxin reductase for the maintenance of high protein synthesis activity in rabbit reticulocyte lysates. Eur. J. Biochem. 131, 303-311.

Jacquot, J.P., Vidal, S., Gadal, P., and Schürmann, P. (1978). Evidence for the existence of several enzyme-specific thioredoxins in plants. FEBS Lett. 96, 243-246.

Jörnvall, H. (1977). Differences between alcohol dehydrogenases. Eur. J. Biochem. 72, 443-452.

Karlin, S., and Altschul, S.F. (1990). Methods for assessing the statistical significance of molecular sequence features for general scoring schemes. Proc. Natl. Acad. Sci. USA 87, 2264-2268.

Kern, R., Malki, A., Holmgren, A., and Richarme, G. (2003). Chaperone properties of Escherichia coli thioredoxin and thioredoxin reductase. Biochem. J. 371, 965-972.

Kuspa, A., Dingermann, T., and Nellen, W. (1995). Analysis of gene function in Dictyostelium. Experientia 51, 1116-1123.
Laurent, T.C., Moore, C.E., and Reichard, P. (1964). Enzymatic synthesis of deoxyribonucleotides. Isolation and characterisation of thioredoxin, the hydrogen donor from Escherichia coli. J. Biol. Chem. 239, 3436-3444.

Maeda, Y., Inouye, K., and Takeuchi, I. (1997). Dictyostelium - A Model System for Cell and Developmental Biology (Tokyo, Japan: Universal Academy Press).

Makino, Y., Yoshikawa, N., Okamoto, K., Hirota, K., Yodoi, J., Makino, I., and Tanaka, H. (1999). Direct association with thioredoxin allows redox regulation of glucocorticoid receptor function. J. Biol. Chem. 274, 3182-3188.

Marcus, F., and Harrsch, P.B. (1990). Amino acid sequence of chloroplast fructose-1,6-bisphosphatase. Arch. Biochem. Biophys. 279, 151-157.

Mazzurco, M., Sulaman, W., Elina, H., Cock, J.M., and Goring, D.R. (2001). Further analysis of the interactions between Brassica S receptor kinase and three interacting proteins in the yeast two-hybrid system. Plant Mol. Biol. 45, 365-376.

Nissen, P., Thirup, S., Kjeldgaard, M., and Nyborg, J. (1999). The crystal structure of Cys-tRNACys-EF-Tu-GDPNP. Struct. Fold Des. 7, 143-156.

Porqué, P.G., Baldesten, A., and Reichard, P. (1970). Purification of a thioredoxin system from yeast. J. Biol. Chem. 245 , 2363-2370.

Russell, D.W., Smith, M., Williamson, V.M., and Young, E.T. (1983). Nucleotide sequence of the yeast alcohol dehydrogenase II gene. J. Biol. Chem. 258, 2674-2682.

Schägger, H., and von Jagow, G. (1987). Tricine SDS polyacrylamide gel electrophoresis for the separation of proteins in the range of $1-100 \mathrm{kDa}$. Anal. Biochem. 166, 368-379.

Stockmann, A. (2002). Analyse der Wechselwirkungen zwischen Thioredoxin 1 und Elongationsfaktor $1 \alpha$. Diploma (MSc) thesis (Kassel, Germany: University of Kassel).

Villeret, V., Huang, S., Zhang, Y., Xue, Y., and Lipscomb, W.N. (1995). Crystal structure of spinach chloroplast fructose-1,6bisphosphatase. Biochemistry 34, 4299-4306.

Wallenfels, K., and Sund, H. (1957). Über den Mechanismus der Wasserstoffübertragung mit Pyridinnucleotiden. Freie $\mathrm{SH}-$ Gruppen und Aktivität bei Alkoholdehydrogenase aus Hefe. Biochem. Z. 329, 17-30.

Wetterauer, B., Jacquot, J.P., and Veron, M. (1992a) Thioredoxins from Dictyostelium discoideum are a developmentally regulated multigene family. J. Biol. Chem. 267, 9895-9904.

Wetterauer, B., Veron, M., Miginiac-Maslow, M., Decottignies, P., and Jacquot, J.P. (1992b). Biochemical characterisation of thioredoxin 1 from Dictyostelium discoideum. Eur. J. Biochem. 209, 643-649.

Yamanaka, H., Maehira, F., Oshiro, M., Asato, T., Yanagawa, Y, Takei, H., and Nakashima, Y. (2000). A possible interaction of thioredoxin with VDUP1 in HeLa cells detected in a yeast two-hybrid system. Biochem. Biophys. Res. Comm. 271, 796-800.

Yang, F., Demma, M., Warren, V., Dharmawardhane, S., and Condeelis, J. (1990). Identification of an actin-binding protein from Dictyostelium discoideum as elongation factor $1 \alpha$. Nature 347, 494-496.

Received August 3, 2004; accepted October 9, 2004 